Voix et Images

voixetimages

\title{
Héloïse : la mort dans cette chambre
}

\section{Lilian Pestre de Almeida}

Volume 7, numéro 3, printemps 1982

Anne Hébert

URI : https://id.erudit.org/iderudit/200342ar

DOI : https://doi.org/10.7202/200342ar

Aller au sommaire du numéro

\section{Éditeur(s)}

Les Presses de l'Université du Québec

\section{ISSN}

0318-9201 (imprimé)

1705-933X (numérique)

Découvrir la revue

\section{Citer cet article}

Pestre de Almeida, L. (1982). Héloïse : la mort dans cette chambre. Voix et Images, 7(3), 471-481. https://doi.org/10.7202/200342ar d'utilisation que vous pouvez consulter en ligne.

https://apropos.erudit.org/fr/usagers/politique-dutilisation/ 


\title{
Héloïse: \\ la mort dans cette chambre
}

par Lilian Pestre de Almeida, Université Fédérale Fluminense, Brésil

\begin{abstract}
«Aimez qui vous aima du berceau dans la bière: Celle que j'aimal seul m'aime encor tendrement. C'est la Mort - ou la Morte.
\end{abstract}

Nerval, "Artémıs", in Les Chimères

Le dernier livre publié d'Anne Hébert, Hélö̈e (Seuil, 1980) est un récit elliptique sur le fantastique des coins de rues. Il diffère à première vue de ses autres cuvres en prose, les récits du Torrent, les romans Les Chambres de bois, Kamouraska, Les Enfants du sabbat dont l'action se passe au Québec. Dans Héloise, le pays natal de l'écrivain n'apparait que dans une brève allusion au milieu d'une page, un voyage de noces offert par une vieille tante que le jeune marié refuse ${ }^{1}$.

Anne Hébert avoue du reste une certaine inquiétude devant l'accueil que ses lecteurs québécois pourront faire à son dernier récit : a Le cadre de la plupart de mes romans se situe au Canada, et mon public est davantage québécois que français. Je crains un peu leurs réactions devant Héloise, dont l'action se passe uniquement à Paris" ${ }^{2}$. Mais ses lecteurs seront certainement sensibles au fait qu'Héloise renoue de façon éclatante avec des thèmes obsédants de l'œuvre hébertienne, en particulier avec la figure inquiétante de la jeune fille maigre $^{3}$

La ville de Paris est évoquée de façon obsédante dans ce court récit d'une centaine de pages. Rues du Quartier Latin (rue Git-le-Cœur, rue de la Huchettel, le Jardin des Plantes, cafés, place Maubert, place de l'Odéon, Jardin de Cluny, un certain appartement près du Bois, dans l'impasse des Acacias. Et surtout le Métro, couloırs, tunneis, rames, bouches, escaliers, affiches lacérées, stations fermées (Cluny, Croix-Rouge). Le récit s'ouvre d'ailleurs par la description minutieuse d'un appartement au charme désuet et suranné. près du Bois, qui attend Christine et Bernard, les fiancés, sans qu'ils s'en soucient (p. 12). L'immeuble 1900 est marqué par trois figures: au-dessus du grand portail d'entrée, “une tête de femme à la chevelure défaite" (Méduse?); dans le salon de l'appartement " un buste de plâtre représentait une 
créature sévère, ni homme ni femme, l'air absent" (homme châtré ou Mère phallique?) et «un bronze représentait Orphée jouant de la lyre, au milieu des bêtes sauvages, pâmées» (p. 11). Et si la fonction du narrateur était celle-là mème de dompter les bētes sauvages (isomorphes de la femme à la chevelure défaite et de la mystérieuse créature ni homme ni femme), prêtes à le dévorer s'il arrête son chant? Orphée grâce à la magie de sa parole obtiendra des dieux infernaux d'exorciser la peur et de jouir de l'interdit, celui de revoir la morte, pourvu qu'il ne la regarde pas de face. Le destin des deux jeunes époux sera autrement tragique: ils seront attaqués dans l'appartement près du Bois par des morts-vivants. Bernard aura la gorge tranchée, Christine sera violée et assassinée après un combat sauvage dans la cuisine. Les agresseurs sont-ils des vampires? C'est vite dit. Des indices semblent pourtant le confirmer: Héloïse et Bottereau ne peuvent sortir du Métro avant la tombée du soir, ils s'alimentent de sang (humain de préférence), les glaces ne réfléchissent pas la beauté osseuse d'Héloïse, ils couchent au Père Lachaise. Mais la trame fantastique des morts-vivants masque autre chose.

Reprenons le récit. Après la description de l'appartement mystérieux. dans une impasse près du Bois, le narrateur évoque au chapitre 2 le déjeuner de fiançailles dans la blonde lumière de la Loire. "Jardin fleuri, longue maison de pierre blanche. Les parents de Christine qui menaient grand train étaient aussi simples et joyeux que leur fille» (p. 13). Mais un fantôme se presse au bout de la table dans la lumière angevine. "La petite silhouette noire et frèle" de la mère (morte) du fiancé lui apparaît. Lui seul la voit. "La voilà au bout de la table qui toque de son dé d'argent sur la nappe pour réclamer la parole" (p. '14). Car elle était couturière de son état. Elle «taillait" et cousait. "Des centaines de robes, de manteaux et de costumes». Bernard se souvient d'avoir grandi dans le «ronronnement de la machine à coudre", les chiffons, "les aiguilles renversées, fuyantes comme du mercure». Enfant tranquille ("ne touche pas», «tiens-toi tranquille»), immobilisé et empêché "par mille petıts fils invisibles, cousus par sa mère, à mème sa peaun (p. 14). Mais le fantôme disparaît. Christine, la fiancée, parle: Bernard a trop bu. Christine est la lumière et la vie. Elle "est le contraire d'un songe". "Elle est la vie". (Qui donc avait déjà dit: "Je suis la lumière et la viè?).

L'intermède angevin est clos. Les fiancés reviennent à Paris par le traın. Ils ressemblent à des frères jumeaux. « Jeans et pull-overs. Hanches étroites chez la fille comme le garcon» (p. 17). Unisexe. A la gare d'Austerlitz ils prennent le Métro. Ils sont heureux. Ils se donnent rendez-vous pour ce même soir. Mais Bernard entend une voix féminine qui chante: "Celle qu'on n'attendait pas/Sort de l'ombre» (p. 20). Bernard s'en défend par une litanie dérisoire: "Bernard aime Christine. Christine aime Bernard". Mais quand il aperçoit enfin Héloïse, maigre, osseuse, pâle, d'une beauté inquiétante, la petite Christine disparaît frappée d'inanité. Le mariage se fera. mais Bernard n'aime plus Christine. Avant le mariage. les fiancés résilient le bail de leur studio clair et nu, rue du Commandeur ${ }^{4}$. Gràce à un personnage entrevu 
à côté d'Hélö̈se dans le Métro, ils prennent un appartement 1900 près du Bois. Celui qui les « attendaịt » dès le début et dans lequel Christine se sentira toujours l'intruse (p. 61). Parce qu'il a vu Héloïse aux cheveux noirs, Bernard ne peut plus supporter la jeune fille claire qu'il avait naguère choisie. Qu'elle touche Bernard, ce contact physique le révu/se. «Christine a pris la main de Bernard qui ne semble pas s'en apercevoir, alors que tout son être se révulse au contact de cette main chaude et douce» (p. 23). Au fond de son cœur, il ne désire qu'une chose. "L'absolue vérité qui se cache au fond là où aucun mensonge ni pitié ne peuvent subsister. Répudier Christine»(p. 40). II découvre avec malaise qu'elle aime les éclairages trop violents, qu'elle est trop bruyante, trop éclatante, qu'elle aime manger et boire.

Le couple de jumeaux apparemment sans histoires aussitôt après leur mariage devient couple étrange. Christine sort tous les jours, va a l'Opéra, travaille, répète et danse. Bernard se terre a la maison, se contentant de cuisiner des plats insipides. Tous les paniers que Christine apporte du marché sont déballés, triés. "Tout ce qui a trop d'odeur ou de goút est mis de côté. Impitoyablement. Par Bernard» (p. 62). "Je suis fidèle à ma promesse de faire la cuisine, se répète-t-il pour se rassurer, tandis que la pensée d'Héloïse le tourmente, de plus en plus" (pp. 62-63). Si par hasard ils sortent ensemble, Bernard est au bord de l'écceurement devant Christine qui mange, boit et rit (cf. chap. 8). Elle aime la viande, le gigot, l'all, le vin. Dans sa claustration. Bernard trompe, en rêve, sa jeune femme avec Héloïse. "Vivre avec l'image renouvelée d'Héloise (...) Ne pas quitter Christine puisqu'il s'est marié avec elle, mais s'émouvoir en secret sur un visage aux pommettes dures, aux yeux en amande. La revoir bien vite. Retrouver au plus tôt sa maigreur souveraıne. Tromper Christine en rêven (p. 63). Et Christine elle aussı se leurre. "La nuit. Dans l'obscurité profonde Christine croyait retrouver Bernard. (...) Comment pouvait-elle se douter que l'homme amoureux contre son flanc s'adressait à une autre femme en rêve et que les ténèbres étaient propices à l'illusion?m (p. 65).

Que le couple rêvé Bernard/Héloise renvoie à Abélard/Héloise c'est trop évident pour qu'on $y$ insiste. Mais le rappel des amants parfaits et impossibles (l'impossibilité prenant source dans la différence de classes, la castration et le sacré) masque un interdit autrement fort. Héloïse est morte, ou, si l'on veut, une morte qui accomplit avec Bottereau "la loi de la terre": les morts mangent les vivants, s'alimentent de leur sang. Bernard découvre peu à peu, dans l'horreur et la fascination, l'identité d'Héloise: elle le blesse tout d'abord au poignet faisant couler du sang (chap. 26 et 28); plus tard, elle lui tranche la jugulaire quand il l'attend au lit pour faire l'amour (agression brutale qu'on prendra pour une tentative de suicide de Bernard que sa femme vient de quitter): enfin, elle le fait mourir au Père Lachaise. Après la découverte du cadavre de Christine dans sa cuisine, Bernard sachant désormais ce qu'il risque, recherche Héloïse dans le métro, un dernier soir, un revolver dans sa poche. Il la retrouve enfin au bout de la nuit et n'ose lever son arme. Châtré 
symboliquement par la «fille maigre», Bernard la suit. «Piéta sauvage», elle l'embrasse et le tue. Mais avant, Bernard lui murmure: « Je t'aime, je t'aime *. Héloïse le cajole avec des mots simples: "Ce n'est que la fascination de la mort, mon chéris (p. 123). Or, cet attrait de la mort, avoué, explicité, occulte en fait l'identification d'Héloise avec la mère, la couturière qui manœuvrait ciseaux et aiguilles.

La médiation de Christine a échoué. La mère, disparue du déjeuner de fiançailles grâce à la parole de la fiancée (Bernard a trop bu), est revenue. Sous une autre forme. Autrement attirante et puissante. Revenons encore à la très courte scène des fiançailles. Lorsque le fantôme de la mère disparait. Bernard est soulagé. "Je suis sauvé, pense Bernard, en serrant très fort la main de Christine dans la sienne» (p. 14). Sauvé de qui? De la mère qui l'a empêché, qui a immobilisé son corps " par mille petits fils invisibles, cousus (...). à même sa peau, quand il était enfant et dormait dans son petit lit. contre le grand lit maternel» (p. 14). Ce lit où il n'y a que la mère (la couturière est une femme xabandonnée par son mari) est de toute évidence isomorphe du ventre et de la tombe.

Christine a coupé la parole à la mère, sans même s'en apercevoir. Elle a le corps souple et fort. Elle est coryphée à l'Opéra (p. 14). Ce mot masculin vient du grec Koruphaios, de Koruphê "la tête", " chef du Chœur dans les pièces du theâtre antique»: "celui qui tient le premier rang dans une partie». Pauvre Christine, coryphée dérisoire. Elle coupe la parole à la couturière, mais restera impuissante devant Héloise. Ignorant la mère mais soupconnant le danger d'Héloise (qu'elle ne rencontrera jamais), elle tente maladroitement de sauver Bernard des aiguilles et des ciseaux de l'une et de l'autre ${ }^{5}$. Émouvante dans sa maladresse et sa légèreté. Bernard, sauvé de justesse après avoir eu la gorge tranchée, pense à l'hòpital: «Maintenant que l'identité d'Héloise ne fait plus de doute, la fuir comme la mort. (...) Après l'épouvante, si des choses bonnes et douces peuvent encore être vécues c'est du côté de Christine qu'il faut établir son espérance. parelle a une ville fortifiée. Si le salut existe quelque part au monde, il ne peut venir que de la petite épouse fidèle, confrontée à un destin trop étrange pour elle. Agir comme sı l'amour était encore possıble entre Christine et lui» (p. 110).

Mais Christıne, "la petite épouse fidèle". de temps à autre, revêt elle aussi les caractéristiques de la féminité menaçante. Après la sortie de Bernard de l'hôpital, Christine prépare un déjeuner: lapin à la moutarde, salade. melon, reblochon, vin. C'est dans son "travail de bouchère"., tranchant et découpant un grand lapın mort, qu'elle sera surprise par son assassin. Et ce cadavre d'animal dépecé préfigure sa mort à elle dans les mains de Bottereau. Bernard en rentrant aécrase du pied le petit cœur du lapin" dans une cuisine qui porte partout «les traces d'une lutte sans merci» (p. 117) avant de découvrir Christine morte. Bien qu'elle mange et puisse a l'occasion manier le couteau pour dépecer un animal abattu, Christine n'est pas mère. Elle est trop jeune fille, trop claire, avec son corps de danseuse tout en jambes 
et sans hanches pour être inquiétante. Elle est plutôt le double de Bernard. voulant vivre.

II y a sans doute dans le récit un épisode prémonitoire et symbolique au sujet de Christine. Un soir, celui de la mort du jeune daneau saigné à blanc au Jardin des Plantes, Héloïse entraine Bernard jusqu'à la rue Gît-leCour, dans une cave profonde, bondée. "Des gens silencieux et figés sont assis autour des tables, éclairées à la bougie. Les visages sont étranges, trop apprêtés et maquillés: ( $p$. 79). Bernard remarque que tout le monde autour de lui boit du bloody-mary. "ll y a des petites lueurs couleur de sang qui clignotent dans chaque verre" (p. 80$)$. Le spectacle qu'on y donne est étrange: les danseuses de can-can, habillées de noir et blanc, portent des culottes d'un rouge vif. Elles ont l'air de poupées et à la fin de la danse frénétique. au moment du grand écart, elles "ne se relèvent pas et demeurent sur le sol, comme des pantins disloqués» (p. 80). L'image, inquiétante, annonce déjà Christine vidée de son sang dans sa cuisine. Le cöryphée de l'Opéra deviendra pantin disloqué dans la cave aux vampires. D'ailleurs, dans la scène de mise à mort de Bernard dans le cimetière. Christine est présente, tenue par Bottereau, "dépenaillée et complètement indifférente» (p. 124).

L'échec de Christine qui devrait apporter la nouvelle loi (l'homme doit quitter père et mère pour faire un avec sa femme) fait que le destin de Bernard est, en définitive, tracé par deux femmes terribles, la mère couturière et la vampire Hélö̈se, qu'il aime et craint, qui l'immobilisent l'une avec ses fils dans un petit lit, l'autre dans ses bras au cimetière; la femme compagne, vite répudiée, n'est pas objet de désir. La passion relève de deux mortes. Lachésis et Atropos: Bernard passe, comme dans un rêve, de la fileuse qui préside a la naissance à la fileuse qui coupe le fil. Cloto, la dévideuse, ne l'intéresse pas: Christine n'est qu'un intermède sans importance et disparaìt devant celle qu'il faut choısir, la fille maigre. Christine n'est qu'un espoır vite conçu et aussitót déçu de vie. L'illusion n'a duré que six mois: du moment où ils se sont connus juśqu'aux fiançailles.

Bernard n'a pas connu son père. Sa mère est une "femme abandonnée" qui a élevé son fils seule. Dans tout le récıt. le seul personnage masculin en dehors de Bernard est Xavier Bottereau, qut accompagne Héloise dans le Métro. Bernard pense d'abord qu'Héloïse est sa fille, mais Bottereau le détrompe: "Héloïse n'est pas plus ma fille que la vôtre, jeune homme. Simple rencontre sous terre. Les hasards du métron (p. 54) ${ }^{6}$. Pour Bernard, autant Héloise l'attire, autant Bottereau lui répugne. Qui est-il? On serait tenté de répondre l'agent immobilier/immobilisant. Adjuvant d'Héloise, soumis comme elle à la "loi de la terre", il agit pour elle, récupère ses vieux meubles aux Puces, arrange l'appartement près du Bois. le loue. C'est lul qui aidera à fixer Bernard dans l'appartement. II est à certains égards un personnage féminısé: petit, vieux, asthmatique, portant toujours un chapeau melon? Son nom d'ailleurs indique un rapport à l'eau féminine (Bottereau). C'est lui qui assassinera sauvagement "l'agneau" 8 Christine, car ce qu'il veut c'est 
du sang frais et généreux. Chez lui, il n'y a aucune tendresse pour les victimes. De la gourmandise pure. Le désir brutal et nu. II n'essaie pas de séduire; a Je viole et je tue ( $p$. 107). Il représente l'instance maternelle dans toute sa férocité sans aucune douceur. II est la face sans pitié de la Mère dévoratrice et maléfique. L'absence du Père dans le récit est encore récupérée, car le lieu où dorment chaque soir, côte à côte. Héloïse et Bottereau, l'endroit où Bernard revient enfin au giron maternel, est le Pere Lachaise. Les derniers mots du récit sont les suivants:

* Le brouillard monte à nouveau, s'étend sur toute l'assemblée. On peut lire le nom de la station Père-Lachaise à travers la brume» (p. 124).

Héloise comporte encore, comme son emblème mystérieux, un texte écrit par Bernard en état second, dans un cours de Droit a la Sorbonne, juste après le choc de la première rencontre avec la fille maigre dans le métro. Au chapitre 5, des notes griffonnées à partir des associations libres sont en fait le compte-rendu synthétique et prémonitoire du destin de Bernard. Nous les reproduisons:

- Et c'est alors que le mort saisit le vif!

On peut lire en outre sur le bloc de Bernard:

"Tout homme trouvé en état d'ivresse sera mis en état d'arrestation. Qui a bu boira. Dent pour dent, œil pour œil. Son œil sombre, ses dents blanches. La fille aux cheveux de nuit s'est volatisée. Bien fin qui la trouvera. Rien ne sert de courir il faut partir à temps" (p. 27).

Aussitôt griffonnées, ces notes sont perdues par Bernard qui bondit "hörs de la salle de cours, comme un chat qui a entendu un oiseau " (pp. 28-29). Nous savons à quel point l"oiseau est lié a la mort chez Anne Hébert ${ }^{9}$. Celu qui a entendu le chant de la mort et l'a désirée, sera puni. Mais la punition permet l'accomplissement du désir incestueux du Fils pour la Mère. Là, Christine est médiatrice à son insu. C'est son viol et son assassinat qui inciteront Bernard à prendre encore une fois le Métro pour se venger, tuant. prétend-il, celle qu'on ne peut tuer, car on ne meurt qu'une fois et Héloïse (comme la couturière) est déjà morte. "Et c'est alors que le mort prend le vif". Celui qui croyait prendre (le chat) est pris (par l'oiseau); celui qui voulait tuer est tué. La première phrase griffonnée dans un cours de Droit se réalise. La mère phallique prend le fils dans ses bras. Celui qui a bu du bloody-mary dans une cave de la rue Gît-le-Cœur est mis en état d'arrestation. Définitive. Car «qui a bu boira», sentence ambiguë et polysémique renvoyant à la fols a Bernard (qui a bu le lait maternel) et à Héloïse. Celle-ci qui a bu du sang et du bloody-mary, dira à Bottereau: "Soyez tranquille, je vous ramènerai ce garçon. Le désir que j'ai de lui est si fort que je boirai sa vie jusqu'à la dernière goutte» (p. 107). «Dent pour dent, ceil pour œil»: la loi de la terre s'accomplit et Christine, personnage dérisoire et christique, "agneau", est vaincue. Devant la jeune fille maigre entrevue dans le métro. Bernard est fasciné par "son cil sombre», "ses dents blanches": trou et bouche d'ombre de l'ogresse originelle. 
Relisant le récit, nous nous apercevons qu'il existe une sorte de complicité secrète, jamais explicitée, entre Héloïse et plusieurs vieilles femmes. Une vieille dame interpelle Bernard dans le Métro et Héloïse peut disparaître sans qu'il s'en aperçoive:

(...) La vieille dame lâche son poteau, agite ses petites mains, comme des serres contre son visage.

- C'est à la station Croix-Rouge que la demoiselle est descendue. Je l'ai très bien vue.

Un homme en bras de chemise proteste avec indignation.

- Tu radotes, la mère, la station Croix-Rouge est fermée depuis longtemps. (chap. 19, p. 73)

Mieux encore, Hélö̈se ne semble s'entendre qu'avec des femmes âgées. L'une de ses amies, Mélanie, tient le lavatory du métro Maubert-Mutualité. C'est « une femme âgée, perruque rousse, vaste tablier amidonné, maquillage théâtrals; elle aide Héloise à nettoyer une tache de sang sur sa manche. C'est d'ailleurs en sortant de ce lavatory qu'Héloise pique Bernard au poignet. avant de lui donner rendez-vous le lendemain dans l'appartement du Bois. II y a peut-ètre même une complicité sournoise entre Héloise et de vieilles parentes à Christine. C'est bien sa'grand-mère ou tante Marthe qui a remplacé les draps de couleur choisis par la jeune mariée par du linge blanc (p. 58). $C^{\prime}$ est encore cette tante Marthe ${ }^{10}$ qui, sous prétexte de maladie. demandera à Christine de venir la voir, permettant à Bernard de reprendre le Métro et d'y retrouver Héloise. Or, il y a là une surdétermination du graphème $M$ (de mère, grand-mère, Mamıe, Marthe, Mélanie)" Toutes ces vieilles femmes ou ces femmes en rouge (Mélanie porte une perruque rousse, les danseuses de can-can des culottes rouges, Héloïse 12 est vêtue de soie rouge le soir où Bernard a la gorge tranchée) aident obscurément celles qui présıdent au destin de Bernard et celui-cı pourra former, grâce à elles, le couple interdit. Bernard/Héloise: le vivant/la mort(e), le Fils/la Mère.

La distance dans le temps ${ }^{13}$ fait d'Héloïse une femme jeune mais d'une autre génération. Avec elle Bernard réalise son rêve secret d'union avec la Mère restée jeune. La source du plaisir chez le lecteur vient de la représentation interdite de l'inceste avec la Mère, "loi de la terre", à l"accomplissement de laquelle il $y$ a complicité sournoise et du fils et de toutes les vieilles femmes. Héloïse est donc l'instance maternelle dans ses trols faces: celle de la génératrice qui donne tout (son sang, son lait, la vie), l'objet du désir et la donneuse de Mort. Son visage fascine Bernard. II s'asseoit “ médusé»" au bord du lit quand elle vient chez lui (p. 97). Elle est Atropos, l'inexorable. "Le visage d'Héloïse, lisse comme un caillou. La beauté des os visibles à travers ses mains translucides. Une flamme mauvalse dans ses yeux couleur de plomb” (p. 99).

Le concept de l'inquiétante étrangeté (das Unheimliche) s'apparente. comme on le sait, à ceux d'effroi, d'angoisse, de peur. C'est "cette sorte de 
l'effrayant qui se rattache aux choses connues depuis longtemps, et de tout temps familières ${ }^{14}$. La ville de Paris, les sculptures lunaires de Cluny, les stations de Métro, la mode rétro, la fontaine de la Place Saint-Michel, le Bois. le Père-Lachaise etc., tout cela nous est connu. Et l'inquiétante étrangeté se révélera d'autant plus, nous semble-t-il, que la familiarité du lecteur avec Paris est grande. L'exemple même du récit serait un cas d'inquiétante étrangeté par excellence, celui où l'on doute qu'un être en apparence animé ne soit vivant (Héloïse, Bottereau, les danseuses de can-can, etc.) et inversement qu'un objet sans vie ne soit en quelque sorte animé (le Métro, l'appartement). II est évident que le sentiment de l'inquiétante étrangeté est inhérent à Héloïse et à Bottereau tout d'abord, ensuite à tous les personnages entrevus (Mélanie, tante Marthe, vieille dame du Métro) et aux lieux mêmes (l'agence de Bottereau, l'appartement, le Métro). Les seuls personnages y échappant au départ sont Bernard et Christine, un couple de jeunes d'aujourd'hui, au bonheur apparemment sans histoires. Nous sommes a la fois dans la vie réelle et dans un univers fantastique où les objets prennent vie, où les morts boivent le sang des vivants...

Pourquoi Hélöise intervient-elle au moment où Bernard vient de se fiancer, de choisir une compagne pour la vie? Pourquoi sa présence frappe-telle d'inanité Christine? Pourquoi Bernard devant Bottereau et Héloise devient-il aussitôt si agressif vis-à-vis de Christine? Ces tratts semblent arbitraires si l'on oublie la relation de désir entre le Fils et la Mère. Bottereau et Héloïse, le couple du Métro, représentent l'image de la Mère décomposée. grâce à l'ambivalence, en ses deux contraıres: Bernard éprouve de la répugnance pour Bottereau et une attirance invincible pour Héloise. Bottereau viole et massacre : Héloïse tue amoureusement. À ces deux images de l'instance maternelle correspond un dédoublement de l'enfant, à la fors Bernard et Christine, celle-ci voulant vivre. l'autre revenir à la Mère.

Bottereau et Héloïse couchent côte à côte dans des tombes voisines au cimetière : celle d'Héloïse est marquée par une ombreile et celle de Bottereau par un chapeau melon. Ces objets restent ambigus dans les deux cas renvoyant à la fois a la féminité et à la masculinité. Le jeune homme, fixé à la mère par le désir de l'inceste, devient incapable d'éprouver de l'amour pour la femme, car il n'arrive pas encore à la séparation des sexes : la Mère est à la foıs la mère et le père (Héloise et Bottereau) et lui-même forme avec Christine un couple unisexe. Leurs habits semblables suggèrent leur identité profonde. La scène où Bernard et Christine font l'amour pour la première fois est révélatrice. Pénétrant dans la chambre de Christine, rue Cardinal Lemoine, Bernard s'émeut devant ses chaussons suspendus au-dessus du lit étroit, qui luı semblent porter des traces de blessure. Les chaussons de son double renvoient a son complexe de castration, ce n'est donc pas par hasard que le nom de Bernard rappelle Abélard. Cet attendrissement de Bernard devant les chaussons de Christine s'explique encore sans doute par l'impression, nonexprimée, mais présente qu'elle n'est pas la Femme inquiétante et phallique et peut le protéger de sa fascination pour la Mère terrifiante (tout d'abord la 
couturière et ensuite la vampire). Elle l'attire dans la mesure où elle porte les traces d'une blessure; mais la découverte de l'appétit de Christine qu। mange et boit l'inquiète au plus haut point. Elle, qui était son double clair, devient pour Bernard l'Autre. Celle-ci en choisissant pour vivre un studio clair et nu, "un lieu flagrant et nul», nie le retour désiré au ventre-tombeau (p. 42). D'ailleurs, l'inquiétude de Bernard devant l'appétit de Christine rappelle en quelque sorte une sorte d'horreur cachée de l'Ogresse qui annule son émotion devant les petits chaussons de la danseuse. "Ces petits chaussons surtout attendrissaient Bernard. II les prit dans sa main et les examina longuement. Les traces d'usure le touchaient comme des blessures" (p. 15). Quelle est cette trace d'une blessure, sinon le rappel occulte d'une castration subie? L'image de Christine oscille donc pour Bernard: son double voulant vivre, compagne solaire, refuge dérisoire contre la Mère, Autre soulevant le dégoût.

Cherchons encore d'autres exemples d'inquiétante étrangeté. Dans Héloise, on rencontre encore le thème du pantin animé. Les danseuses de can-can sont-elles des poupées ou des danseuses? Des machines articulées ou des mortes vivantes? Nous retrouvons lá tout ce qui touche au thème du double: on y voit apparaitre des personnes qui, vu la similitude de leur aspect. pourraient être considérées comme identiques: Bernard et Chrıstine au début du roman, le coryphée de l'Opéra se multipliant en un nombre indéterminé de pantins animés. Mais chez Bernard se développe, à partir même de sa première rencontre avec Héloïse, une instance particulière qui critique sans cesse Christine et toutes les autres fémmes qui ne sont pas Héloise, c'est-à-dire la mère morte. Et cette instance crée une distanciation dépréciative: aux yeux de Bernard, Christine est tour à tour "ridicule" avec ses sabots, "répugnante" dans son appétit, "énervante». Une amie rencontrée au hasard au café est "grotesque" parce qu'elle porte le long sans avoir la grâce de la fille maigre. Il passe son temps à dévisager les femmes dans la rue: "toutes ces silhouettes de femmes entrevues, leurs parfums qui le frôlent, parfois la surprise de leurs volx, trop sonores et fortes, l'oppressent et l'épuisent "(p. 33). Mais nous savons maintenant quel est le sens de la quête de Bernard parmi les mille et un visages fémınins de la foule anonyme.

L'angoissant ici, dirait Freud, "est quelque chose de refoulé qui se montre à nouveau " et l'inquiétante étrangeté "n'est en réalité rien de nouveau. d'étranger, mais bien plutôt quelque chose de familier, depuis toujours, à la vie psychique, et que le processus de refoulement seul a rendu autre"15. En rejetant son double qui veut vivre, Bernard confirme symboliquement sa castration par la Mère phallique et se réunit à elle dans une tombe-ventre du Père-Lachaise.

L'appartement du Bois était, nous l'avons vu, un lieu hanté. Mais après la mort de Christine sous le couteau de Bottereau, son charme est évacué. désaffecté. Et. pour la première fois, Bernard y étouffe:

"Bernard ouvre la porte. Le silence le prend à la gorge. Tout dans l'appartement semble s'être définitivement arrèté comme une vieille horloge. 
L'air que Bernard respire est raréfié. Les murs n'ont plus aucun écho. Les meubles et les tentures se sont ternis. Chaque objet est vieux, usé, irrémédiablement fini, perdu. La vie ici n'a que faire. Jamais plus Bernard n'habitera ces lieux désaffectés. II étouffe $*$ (p. 117).

Or, cela nous permet de comprendre que l'appartement "attendait " Christine aussi. Celle-ci, double de Bernard voulant vivre et s'opposant à l'instance maternelle, rejetait l'appartement parce qu'il était préparation pour la mort. Cet appartement, reconnu par Bernard dès le premier instant, était isomorphe du corps maternel auquel il retourne en mourant dans les bras d'Héloise, entouré par la foule des morts qui se rapprochent du couple interdit Mère/Fils et «les enferment étroitement» (p. 124). Cette mort inquiétante renvoie au fantasme de la vie dans le corps maternel. "Dans ce cas, l'Unheimliche est ce qui autrefois était heimsch. de tous temps familier"16. Héloise est a la fois "Piéta sauvage et Marie sanguinaire. Bloody Mary ${ }^{\prime}$.

1. La jeune épouse, le jour même du mariage, dit à son mari: “Dommage que tu $n$ 'aies pas accepté le vovage de noces offert par tante Marthe. Un petit tour au Canada, ça $\mathrm{m}$ 'aurait bien plu, moix (chap. 14, p. 57). Mais Bernard ne peut partir. car il a hâte de vivre dans un certain appartement.

2. Les Nouvelles Littéraires, $n^{\circ} 2738$, p. 28.

3. Elle apparait en particulier dans le poème du même nom du Tombeau des rois et dans le personnage de Lia des Chambres de bois.

4. Autre nom symbolique. Les fiancés sont vóués à la mort. Ils abandonnent l'appartement placé sous le signe du Commandeur pour habiter l'appartement marqué par la tête de la Méduse.

5. Il est intéressant de noter que dans tout le récit, Il n'y a aucune allusion aux canines du vampire. Héloise manipule sans cesse des aiguilles (des seringues) et un objet tranchant (rasoir probablement). Avant son attaque contre Bernard, un jeune daneau est trouvé mort au Jardin des Plantes, une large blessure au cou. « Saigné a blanc. (ct. chap. 21, p. 76)

6. Héloise elle aussı dira à Bernard: Bottereau est a un compagnon, un coureur de métro, comme moi. (p. 70$)$

7. Chapeau melon, que Bottereau laisse sur sa tombe quand il rentre au Père-Lachaise. Héloïse, elle, laisse sur la sienne une ombrelle. Nous y reviendrons (cf. chap. 34. p. 108).

8. Bottereau appelle toujours les deux fiancés $\alpha$ mes agneaux (cf. p. 47, 55 et passim.)

9. Une étude de ses poèmes serait très révélatrıce. Voır l'oıseau dans l'ensemble du Tombeau des rois.

10. On ne sait si cette mystérieuse tante Marthe. dont on parte mais qui n'apparait jamais, est en fait la tante de Bernard ou de Christine. Probablement de Christine. car c'est elle ou Mamie qui a changé les draps.

11. Le mème graphème réapparaît dans métro et dans la boisson commandée par Héloïse dans la cave, rue Gît-le-Caur, bloody-mary.

12. «Elle est en grand apparat. (...) Elle laisse tomber son manteau de velours noir par terre. Sa robe de soie rouge, pleine de volants. étoffe sa maigreur. Tout le temps de sa visite, Héloïse conservera ses gants, très longs et noirs* (p. 97).

13. Héloïse s'habille toujours comme un personnage de 1900 .

14. Freud, Sigmund, *L'inquiétante étrangeté», Essais de psychanalyse appliquée. Paris, Gallimard, 1971, p. 165. 
15. Ibid., p. 194.

16. Freud, id., p. $199-200$.

17. Il faudrait revenir encore une fois au texte de Bernard griffonné en état second dans un cours de Droit. Le narrateur décrit le départ précipité du personnage: Bernard bondit h hors de la salle de cours, comme un chat qui a entendu un oiseau * (p. 28-29). Le texte exprime, sous une apparence naïve, le désir de Bernard, qu'on pourrait résumer par la formule le chat veut manger l'oiseau. Le chat (c'est-à-dire Bernard) veut prendre l'oiseau (c'est-à-dire Héloise). En d'autres termes, le chasseur masculin chasse ta proie féminine. Mais ce que le non-dit du texte véhicule de facon allusive c'est la même formule (le chat mange l'oiseau) avec un sens * autren. Les dictionnaires d'argot enregistrent respectivement pour chat et oiseau, « sexe de la femme* et atriste personnage*. D'aitleurs faudrait-il écrire chat ou chas? Le signifiant | $f$ a| de cette formule a autrex renvoie donc a un double signifié ou concept, le sexe téminin et le fond de l'aiguille, instrument de la Mère, de la Morte et de la Mort (d'ailleurs chas vient de capsa, du lat. capsus a coffre ). Que le trajet du beau texte d'A. Hébert sur le couple mystérieux de Bernard/Héloïse puisse se résumer en un jeu de mots (/e chat veut manger /oiseau mais le chat/chas mange ("oiseau) justifie notre approche. Le contenu manifeste du récıt - un couple de vampires attaquant un couple de vivants dans le Paris d'aujourd'hui - masque un rapport à la Mère et pourrait prendre sa source dans un jeu de mots.

Freud nous a montré que le plaisir que nous procure l'humour dérive de l'épargne d'une dépense affective. Il représente une méthode de regagner, sublimé. par le jeu de notre activité psychique (celle de l'écrivain comme celle du lecteur) un plaisir quien réalité le développement seul de cette actıvité nous avait fait perdre. L'esprit et l'humour - encore si mal analysés dans l'ceuvre d'A. Hébert et qu'il faudrait approfondir en partıculier dans l'admırable récit des Enfants du sabbat - est un élément de libération. Du mal. 